\title{
AN IMPROVED RESOURCE RESERVATION ALGORITHM FOR IEEE 802.15.3 ${ }^{1}$
}

\author{
Xin Liu, Qionghai Dai, Qiufeng Wu \\ Dept. of Automation, Tsinghua University, Beijing, China, 100084 \\ Email:liux01@mails.tsinghua.edu.cn
}

\begin{abstract}
In this paper, we propose an improved resource reservation algorithm ESRPT (Enhanced Shortest Remaining Processing Time) for bursty traffic based on IEEE 802.15.3 standard. In this algorithm, each transmitter reports current fragments number of the first MSDU (MAC Service Data Unit) and all of the fragments number of the remainder MSDUs in the pending transmission queue to PNC (piconet coordinator). In the next superframe, PNC firstly allocates part CTAs (Channel Time Allocation) for each stream based on the remainder fragments number of the first MSDU by SRPT rule, then allocates remainder CTAs for each stream based on all fragments number of remainder MSDUs by the same SRPT rule. This algorithm decreases the job failure rates of delay-sensitive video streaming efficiently at the cost of slightly increasing communication overheads. Simulation results show that our proposed ESRPT method achieves better performance in QoS for multimedia streams compared to the existing SRPT schemes.
\end{abstract}

\section{INTRODUCTION}

The wireless link is considered a bottlenecked resource due to the difficulty in effectively allocating the shared resource to provide service guarantees. In IEEE 802.15.3 networks with a shared wireless channel, link arbitration is a core issue for flows with QoS requirements due to the stochastic character of the channel, the network being interferencedominated and the bursty nature of multimedia traffic. In this paper, we propose a simple MAC layer frame scheduling algorithm ESRPT (Enhanced Shortest Remaining Processing Time) to deliver timeliness guarantees for VBR (Variable Bit Rate) traffic in general, and multimedia MPEG-4 traffic in particular, with a centralized MAC mechanism. The centralized controller enjoys privileged access to the channel and is responsible for allocating medium access opportunities to every associated flow. The scheduling algorithm in the centralized controller arbitrates which flow accesses the medium when, for how long and on which logical/physical channels. A key insight of this paper is that our approach is to schedule frames at the link layer only, independent of the details of the application layer and wireless channel.

The rest of this paper is organized as follows. In Section II the network architecture and system description are stated. In section III, existing scheduling algorithms are presented and their drawbacks are analyzed, then detailed description of our ESRPT scheme is presented. Numerical results are compared in section IV. Finally, conclusions are given in section $\mathrm{V}$.

\section{NETWORK ARCHITECTURE AND SYSTEM DESCRIPTION}

\subsection{Piconet Architecture}

The basic component in IEEE 802.15.3 is device (DEV). Two or more DEVs within a personal operating space (POS) communicating on the same physical channel comprise a piconet. One DEV is required to assume the role of PNC (piconet coordinator) of the piconet. The PNC always provides the basic timing for the WPAN. Additionally the PNC manages the quality of service (QoS) requirements, power save modes and access control to the piconet. The basic construct of a piconet is shown in Fig. 1.

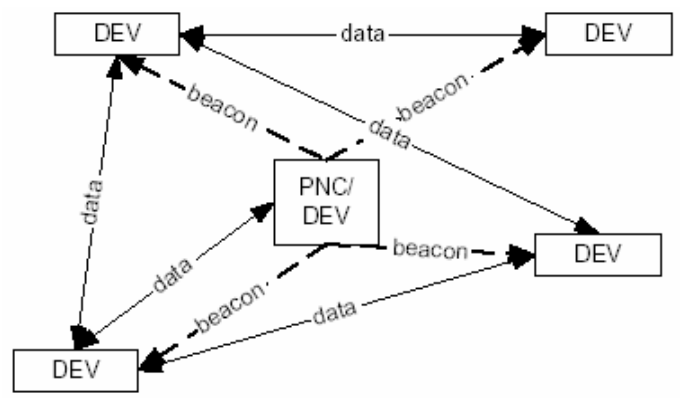

Figure1. 802.15.3 piconet elements

\subsection{Channel Time Management}

Data is communicated directly between source DEV and destination DEV after PNC broadcasts beacon which

\footnotetext{
${ }^{1}$ This work is supported by the key project of NSFC (No.60432030) and Distinguished Young Scholars of NSFC (No.60525111)
} 
including the time allocation of this superframe. Timing in the 802.15.3 piconet is based on the superframe, which is illustrated in Fig. 2 [1].

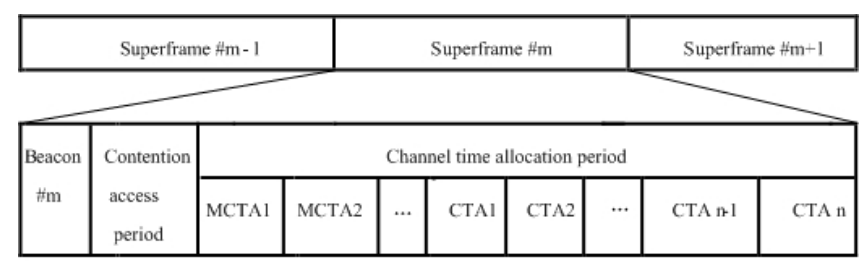

Figure2. 802.15.3 piconet superframe

The superframe is composed of three parts:

The beacon is used to set the timing allocations and to communicate management information for the piconet.

The contention access period (CAP) is used to communicate commands and/or asynchronous data if it is present in the superframe.

The channel time allocation period (CTAP) is composed of channel time allocations (CTAs), including management CTAs (MCTAs). CTAs are used for commands, isochronous streams and asynchronous data connections. The length of the CAP is determined by the PNC and communicated to the DEVs in the piconet via the beacon. MCTAs are a type of CTA that is used for communication between the DEVs and the PNC. The CAP uses CSMA/CA for the medium access. The CTAP, on the other hand, uses a standard TDMA protocol where the DEVs have specified time windows.

\subsection{Channel Simulation Model}

There has been much research to study the wireless channel model to determine the effect of fading on network performance. Wang [2] investigated the accuracy of a firstorder Markov process in modeling data transmission on a Rayleigh fading channel. The simplest example of quantized model is two-level model. Michele [3] shows that a firstorder binary Markov for the success/failure process is a good approximation. In this paper, we try to use two states Markov model to approximately represent the fading channel, as shown in Fig. 3.

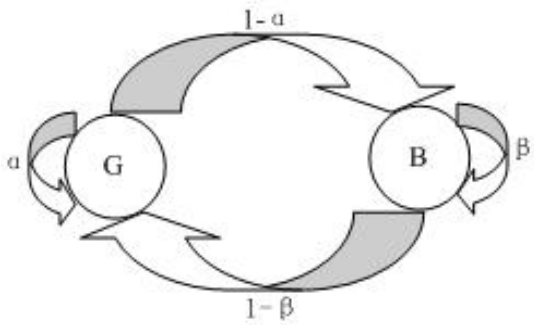

Figure3. Two states Markov model for the fading channel The transition probabilities matrix is given by

$$
P=\left(\begin{array}{cc}
P_{00} & P_{01} \\
P_{10} & P_{11}
\end{array}\right)=\left(\begin{array}{cc}
\alpha & 1-\alpha \\
1-\beta & \beta
\end{array}\right)
$$

"0" stands for good state and " 1 " stands for bad state. We set different Frame Error Rate (FER) in good/bad state. The term "frame" herein means MAC frame called MAC Protocol Data Unit (MPDU) in IEEE 802.15.3 standard. Frame error rate is the effective error rate seen by the MAC after error correction but does not include the effect of retransmissions

\subsection{System Description}

There are several video streams all together within a piconet. In order to handle large data units from layers above the MAC sub layer, IEEE 802.15.3 standard supports the fragmentation and defragmentation of these data units. The fragments are numbered with a sequence number for the upper layer unit (MAC Service Data Unit , MSDU) as well as a sequence number for the fragment (MAC Protocol Data Unit, MPDU) itself. Video stream has deadline because of the decoding limitation. We suppose it is known by source $\mathrm{DEV}$ to save bandwidth. If the time between video frame arriving to source DEV in MAC layer and its transmission out from source DEV exceeds the deadline, source DEV will drop the video frame without informing destination DEV and automatically transmit next video frame. Source DEV detects the deadline limit before transmition each MPDU. If one MPDU is overdue, all of the MPDUs having the same video frame number will be dropped without transmitting to upper layer even if some anterior MPDUs have been transmitted correctly to destination. For the purpose of saving power and simplicity, superframe is generally designed to be fixed. In this way, some DEVs without communication can temporarily sleep for some fixed time and wake opportunely at the beginning of each superframe. Our object is to design a scheduling algorithm of channel time allocation for PNC in each superframe to reduce Job Failure Rate (JFR) as much as possible. JFR is the rate at which video frames are dropped due to missing their deadlines herein.

\section{ALYSIS OF CURRENT APPROACHES AND PROPOSED ESRPT ALGORITHM}

Mustafa Demirhan proposed a SRPT (Shortest Remaining Processing Time) method to momentarily update queue size of each stream by adding a byte to MAC header [4]. With load information from every packet exchange, the PNC is aware of the instantaneous time allocation requirement of each flow, then the PNC can dynamically allocate the idle bandwidth of no load or lowly loaded nodes to the overloaded flows. PNC allocates channel time in the ascending order of the queue size of each flow until there is no idle time remained in current superframe. The detailed process can be described as follows: PNC first allocates channel time to meet the reserved bandwidth requirements. Herein the reserved bandwidth is requested by source DEV 
when registering a flow to piconet. Then the total instantaneous idle capacity can be calculated as the sum of the unreserved bandwidth and the instantaneous unused reserved bandwidth by lowly loaded flows. Next overloaded flows are allocated excess capacity in the ascending order of their overload queue size. All overloaded flows are always allocated at least their reserved utilization.

The idea behind scheduling tasks by discriminating them based on their processing time is well known from queuing theory [5]. The queue size of flows to a large degree stands for the processing time of PNC. By minimizing the number of outstanding requests in a system, Little's Law [5] supports the fact that SRPT minimizes the aggregate mean response time of the system. The object in this paper is to design a proper scheduling algorithm to reduce JFR as much as possible, namely to increase Job Success Rate as much as possible. In a limitary duration we can increase Job Success Rate through enhancing service rate, or reducing mean service time for the jobs having the same deadlines. However, in general the deadlines are not the same for different video frames. That is to say, the limitation of duration is different for each video frame. Therefore it's inapposite for SRPT to consider the queue size of each flow as the whole because the queue consists of a few video frames with different deadlines. The optimized scheduling algorithm should be that PNC firstly sorts all of the video frames based on their deadlines and queues the video frames with the same deadlines according to SRPT rule. The first factor we should consider is the deadline and then the second factor is the queue size. The optimized scheduling method can be described as EDF (earliest deadline first)SRPT and the unit to schedule is video frame or MSDU seen in the MAC layer. Single-handed SRPT is apt to appear such a case: firstly transmitted video frames are from the flow with small queue size while other more exigent video frames from other flows with big queue size are overdue because they are procrastinated to transmit. But it's too complex and infeasible to sort all of the video frames in urgent order since it will increase a lot of overheads in transmission and computation complexity in PNC. To balance the complexity and the performance, we group the video frames having similar deadlines. In general the duration of a superframe is 10 to $15 \mathrm{~ms}$ and the frame arriving interval i.e. deadline interval between the adjacent video frames in the queue is $33 \mathrm{~ms}$. The most urgent mission we should complete is to transmit the first unoverdue video frame in each queue in the next 10-15 ms. As for the posterior video frames whose deadlines are at least $33 \mathrm{~ms}$ from now on, we can handle them during subsequent superframes in the case that there is no idle time slot remained in current superframe. So it's enough for us to divide all of the video frames into two groups: the first video frame and the remainder video frames in the pending queue. For these two groups video frames we adopt SRPT scheduling algorithm respectively and the final CTA allocated to each stream is the sum of the two parts. We can foresee that the performance will be enhanced in ESRPT compared with SRPT.

\section{NUMBERICAL RESULTS}

In this simulation, we do not consider MAC service in CAP mode since it has been shown to not support tight QoS guarantees and distributed service differentiation suffers from unpredictable delays and unfairness [6], [7]. As for the traffic source, we use an MPEG-4 traffic generator that generates traffic which has the same first and second order statistics as an original MPEG4 trace. The MAC layer payload data rate is set to $110 \mathrm{Mbps}, 200 \mathrm{Mbps}$, and 480 Mbps separately and the physical preamble is assumed to be $15 \mu \mathrm{s}$. MAC overheads such as header size, guard times, aFirstCTAGap, SIFS and MIFS are set as regulated in 802.15.3 Draft D17. We utilize first-order two states Markov frame error model to approximately represent the wireless channel. In this simulation we set FER $=0.0$ in good state and $\mathrm{FER}=0.15$ in bad state. The average duration time of good or bad state should be hundreds of milliseconds since the typical channel model is a slow decaying channel in UWB systems. We change channel state between superframes. Superframe size (SFS) is set to fixed $15 \mathrm{~ms}$, and the average duration of channel state is set to 50 slots that are equal to $750 \mathrm{~ms}$. To study the case of multi flows compounding together, we set the interval of different flows is $100 \mathrm{~ms}$. We only consider interactive video traffic such as wireless network conferences and interactive games whose deadline should be less than one hundred of milliseconds. We set deadline of all flows to $60 \mathrm{~ms}$. In order to get results congruous with statistical meaning, we set simulation duration is 800 s including about more than one thousand of average durations of channel state. Max fragment size (payload in MAC layer) is set to $1 \mathrm{~K}$ bytes. All of the results are obtained in NS-2 simulation platform.

From the knowledge of coding and decoding we know that if I frame is lost or wrong, other frames attributed to this GOP will not reconstruct even if other frames are transmitted correctly. Similarly the $\mathrm{P}$ frames also have impacts on $\mathrm{B}$ frames. The GOP of the MPEG-4 model generator is: I, B, B, P, B, B, P, B, B, P, B, B, so we use such a formula to compute the resulting JFR:

$$
J F R=\frac{\frac{12}{12} \times \frac{L_{I}}{N_{I}}+\frac{5}{12} \times \frac{L_{P}}{N_{P}}+\frac{1}{12} \times \frac{L_{B}}{N_{B}}}{\frac{12}{12}+\frac{5}{12}+\frac{1}{12}}=\frac{12 \rho_{I}+5 \rho_{P}+\rho_{B}}{18}
$$

where $L_{I}, L_{P}, L_{B}$ is the lost number of I, P, B frames, $N_{I}, N_{P}, N_{B}$ is the total number of I, P, B frames that source DEV should transmit to destination, and $\rho_{I}, \rho_{P}$, $\rho_{B}$ is the I, P, B frame loss rate. 
At first, we look at the case where bandwidth is $110 \mathrm{Mbps}$, code rate is $6 \mathrm{Mbps}$. Number of flows varies from 2 to 9 . JFR is plotted versus flow counts in Fig. 4. Two different scheduling algorithms are shown for comparison: SRPT and ESRPT. We can see that ESRPT method always obtains the least JFR because ESRPT allocates time for the most exigent video frame firstly, i.e. the first video frame in the queue. In comparison SRPT is likely to ignore I frames with large MPDUs which are more important than the $\mathrm{P}$ or $\mathrm{B}$ frames with small MPDUs. When the flow number is seven in a piconet, JFR of ESRPT method is only $13.6 \%$ while that of SRPT method is $18.4 \%$. When the flow number is small, there is no distinct difference between these two methods since the bandwidth provided is relatively enough, but when the flow number increases, ESRPT method slacks the trend of JFR increasing rapidly with the flow number.

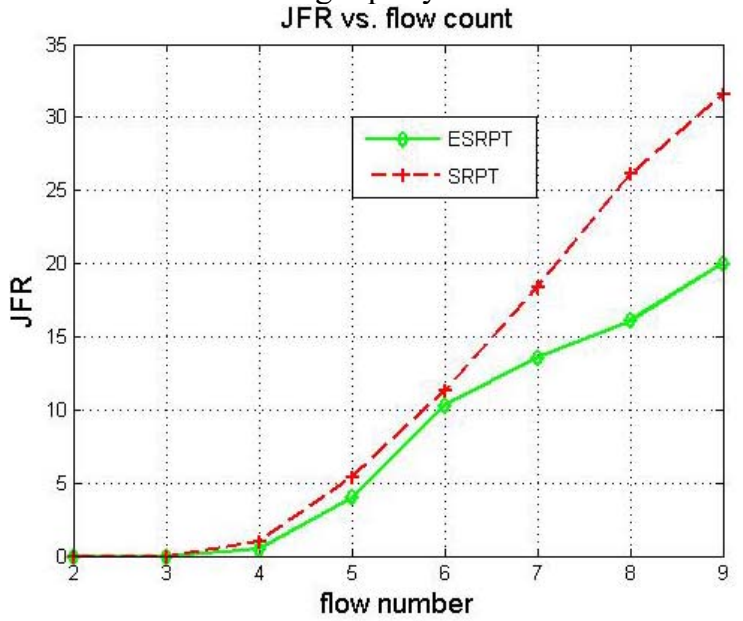

Figure4. Bandwidth $=110 \mathrm{Mbps}$, code rate $=6 \mathrm{Mbps}$, JFR vs. flow count

In Fig. 5, the parameters are the same as in Fig. 4, except that bandwidth is $200 \mathrm{Mbps}$ and code rate is $8 \mathrm{Mbps}$ for each flow. It is obvious that the tendency is the similar with Fig.4.

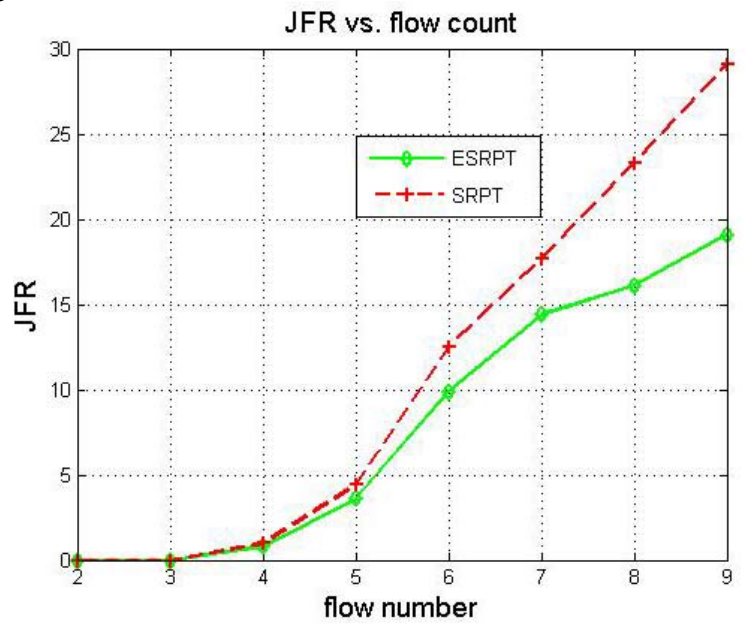

Figure 5 . Bandwidth $=200 \mathrm{Mbps}$, code rate $=8 \mathrm{Mbps}$, JFR vs . flow count

\section{CONCLUSIONS}

In this paper, an improved resource reservation algorithm ESPRT (Enhanced Shortest Remaining Processing Time) based on IEEE 802.15.3 standard is studied in a timevarying wireless slow fading channel environment. A simple two states Markov model has been used: good channel state and bad channel state in which Frame Error Rate is different. The source DEV sends its request to PNC in its own CTA in current superframe, and then PNC is responsible for making channel time allocation decision in the next superframe in terms of a scheduling scheme and broadcasting it in the beacon at the beginning of the next superframe. In our algorithm each transmitter reports remainder fragment number of the first MSDU and fragment number of the remainder MSDUs in the pending transmission queue to PNC separately. In the next superframe, PNC allocates CTAs (Channel Time Allocation) for each stream separately based on the queue information by SRPT rule. In comparison with existing SRPT methods, our ESPPT method always obtains the least Job Failure Rate (JFR) in different parameters cases in simulation NS-2 environment. Our algorithm is effective and simple at the cost of slightly increasing communication overheads, so it is viable for power save mode applications such as WPAN.

\section{REFERENCES}

[1] "Draft Standard for Telecommunications and Information Exchange Between Systems --LAN/MAN Specific Requirements --Part 15.3: Wireless Medium Access Control (MAC) and Physical Layer (PHY) Specifications for High Rate Wireless Personal Area Networks (WPAN)", Draft P802.15.3/D17, February 2003

[2] Hong Shen Wang; "On verifying the first-order Markovian assumption for a Rayleigh fading channel model," Universal Personal Communications, 1994. Record. 1994 Third Annual International Conference on, 27 Sept.-1 Oct. 1994. Page(s): $160-164$

[3] Zorzi, M.; Rao, R.R.; Milstein, L.B.; "On the accuracy of a first-order Markov model for data transmission on fading channels. Universal Personal Communications," 1995. Record. 1995 Fourth IEEE International Conference on, 6-10 Nov. 1995. Page(s): $211-215$

[4] Rahul Mangharam, Mustafa Demirhan, Ragunathan Rajkumar, Dipankar Raychaudhuri, "Size Matters: Size-based Scheduling for MPEG-4 over Wireless Channels," SPIE Conference on Multimedia Computing and Networking 2004, Jan.2004

[5] L.Kleinrock, "Queuing Systems, volume II, Computer Applications,", John Wiley \& Sons, 1976

[6] A. Lindgren, A. Almquist, O. Schelén. "Evaluation of Quality of Service schemes for IEEE 802.11 wireless LANs," LCN 2001

[7] M. A. Visser, M. E. Azrki. "Voice and data transmission over 802.11 wireless network," Proc. of PIMRC'95, pp. 648-652, 1995 